\title{
DESAFÍOS PARA LA GOBERNANZA EN ESPACIOS PERIURBANOS METROPOLIZADOS: EL CASO DE NONGUÉN, EN CONCEPCIÓN, CHILE
}

\section{CHALLENGES FOR GOVERNANCE IN METROPOLITAN PERIURBAN SPACES: THE CASE OF NONGuÉN, IN CONCEPCION, CHILE}

Fecha recepción: 26 de noviembre 2018 / fecha aceptación: 15 de enero 2019

\section{Javier Antonio León Aravena ${ }^{1}$, Felipe Andrés Saravia Cortés ${ }^{2}$ e Ignacio Bisbal Grandal}

\section{Cómo citar este artículo:}

León, J., Saravia, F., y Bisbal, I. (2018). Desafíos para I a gobernanza en espacios periurbanos metropolizados: el caso de Nonguén, en Concepción, Chile. Revista Pensamiento y Acción Interdisciplinaria, 4(2), 24-43.http://doi.org/10.29035/pai.4.2.24

\section{Resumen}

\begin{abstract}
A partir del análisis del caso del sector Nonguén en el área metropolitana de Concepción, Chile, se desarrolla una reflexión exploratoria enfocada en identificar desafíos para la gobernanza territorial de espacios periurbanos de metropolización reciente, basada en las temáticas que surgen como diagnóstico en el diálogo con los actores locales organizados implicados en dicha gobernanza. Se utiliza una metodología cualitativa. Los datos provienen de 9 informes de discusiones grupales de una jornada realizada con dirigentes vecinales del sector. Se aplicó análisis de contenido, usando el software Atlas.ti. Los resultados muestran que la incorporación a la metrópoli genera, entre otras cuestiones, pérdida de vida comunitaria y del estilo de vida asociado a lo rural. Emerge el desafío de mantener lo valorable de lo rural, al tiempo que se avanza en la urbanización. Destaca la necesidad de aumentar el poder vecinal en la determinación del devenir del espacio en que se habita y de regular el desarrollo inmobiliario.
\end{abstract}

Palabras clave: Gobernanza; Territorio; Espacio; Vinculación bidireccional; Universidad.

\footnotetext{
${ }^{1}$ Chileno, Trabajador Social, Magíster en Políticas Sociales y Gestión Local. Doctor en Ciencia Política. Académico del Departamento de Ciencias Sociales, Universidad del Bío-Bío, Concepción, Chile. Email: jleon@ ubiobio.cl

${ }^{2}$ Chileno, Trabajador Social, Magíster en Desarrollo Local y Regional. Doctor en Ciencias Sociales en Estudios Territoriales. Académico del Departamento de Ciencias Sociales, Universidad del Bío-Bío, Concepción, Chile. Email: fsaravia@ubiobio.cl

${ }^{3}$ Español, Arquitecto. Doctor en Urbanismo. Académico del Departamento de Planificación y Diseño Urbano, Universidad del Bío-Bío, Concepción, Chile. Email: ibisbal@ubiobio.cl
} 
Abstract

\begin{abstract}
An exploratory study is developed based on the analysis of the Nonguén case, located in the metropolis of Concepción, Chile. This study aims to answer which are the issues that territorial governance processes of recent metropolizated peri-urban spaces should address, and which are the actors involved in such spatial governance. A qualitative methodology is used. Qualitative data is taken from 9 reports, prepared with information extracted from focus groups with neighborhood leaders. Content analysis was applied, using Atlas.ti software. The results show that the incorporation to the metropolis generates loss of community life and the lifestyle associated with rurality. The emerging challenge is how to maintain the value of rural life while advancing in urbanization. In order to define the future of the living space regulating the real estate development, neighborhood empowerment should be increased.
\end{abstract}

Keywords: Governance; Territory; Space; Bidirectional linkage; University.

\title{
Introducción
}

Este trabajo plantea una reflexión exploratoria acerca del vínculo entre gobernanza territorial y problemas socio-espaciales, en espacios periurbanos de metropolización reciente. Se aborda el caso de Nonguén, una zona periférica del municipio de Concepción, Región del Biobío (Chile), que durante las últimas décadas se ha incorporado a la mancha urbana metropolitana.

El sector Nonguén, posee una población sobre diez mil habitantes. Según datos del Centro de Salud Familiar -CESFAM- en la localidad atienden una población que bordea los 14 mil habitantes (MINSAL, 2017). Nonguén es una cuenca de 44 km2 de superficie que pertenece administrativamente a dos municipios: Concepción y Chiguayante, limitando con la comuna de Hualqui (ver figura 1). Su estero, de tercer orden y $15 \mathrm{~km}$ de longitud, tributa al río Andalién. En su interior alberga la Reserva Nacional Nonguén (de 2931 Ha, declarada en 2009), además de superficie forestal y agrícola, y el sector urbano de Valle y Villa Nonguén, pertenecientes a la comuna de Concepción. Este valle se ha visto sometido periódicamente a fuertes lluvias que han dado lugar ocasionalmente a inundaciones de consecuencias catastróficas. El último episodio tuvo lugar el 10 de julio del año 2006. En este evento, el estero Nonguén y el río Andalién -cuerpos de agua que atraviesan y delimitan el sector-se desbordaron inundando el barrio.

El sector Nonguén tiene una historia amplia y compleja. Destaca su condición rural que hasta los años 50's del siglo XXI pertenecía a pocos propietarios y que cumplía funciones de tipo agrícola alimentario para la ciudad de Concepción. Su desarrollo habitacional se remonta a la década de los 60's, principalmente después del terremoto que asolara a la ciudad, y responde en parte, a la necesidad de expansión y permanente escasez de suelos dentro de esta, gatillada por la migración rural-urbano, a partir del desarrollo del modelo de sustitución de importaciones que hizo que el área de Concepción y alrededores albergara una gran inversión estatal 
Desafíos para la gobernanza en espacios periurbanos metropolizados: el caso de Nonguén, en Concepción, Chile por Javier León Aravena, Felipe Saravia Cortés e Ignacio Bisbal

Grandal.http://doi.org/10.29035/pai.4.2.24

para la instalación de complejos industriales que requerían mano de obra y simultáneamente la expansión de servicios asociados. Este crecimiento industrial en las diversas localidades del área costera de esta región genera las bases estructurantes del fenómeno de conurbación primero y de metropolización en la actualidad.

Actualmente, el sector Nonguén, cumple principalmente la función de zona residencial de capas medias/bajas, y tiene la potencialidad de desplegar otras funciones relevantes que por ahora no se consolidan en una identidad barrial. Tal es el caso de la función educacional universitaria, que con la presencia de la Universidad del Bío-Bío, moviliza un potencial de seis mil personas diariamente en la zona de entrada a Nonguén, a lo que habría que sumar una escuela secundaria industrial que presta servicios al área metropolitana y otra red de centros educativos primarios para la población local. En el sector se han desarrollado también servicios de alimentación, principalmente al interior de Nonguén, con restaurantes que apelan a las tradiciones campesinas y ponen en valor su oferta basados en la cocina tradicional chilena y otros que enfocan su oferta a la población flotante estudiantil. Existen complejos deportivos que no están articulados y otro importante cúmulo de actividades que pueden generar espacios de crecimiento cultural, social, político y económico con un perfil propio del sector. Su entorno y el hecho de ser la vía de acceso a la Reserva Nonguén, produce que el valor del suelo para proyectos inmobiliarios emplazados en zonas de alto valor natural esté subiendo constantemente en una metrópoli con escasez relativa de este recurso, cuestión tensionada por los instrumentos de regulación y la especulación inmobiliaria (Diario Concepción, 2018).

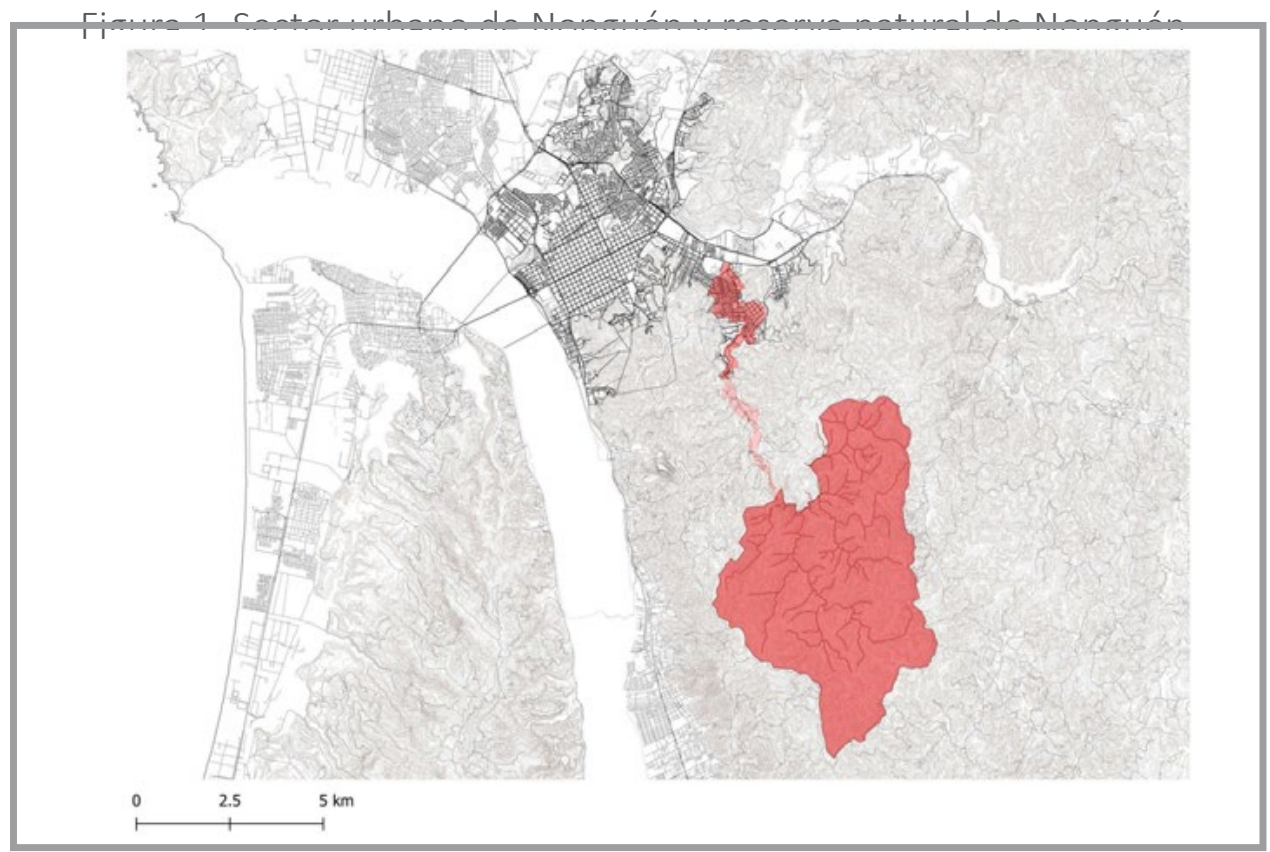

Fuente: elaboración propia 
La reflexión de este trabajo surge en el marco de tres proyectos de investigación, que tienen como una de sus características la vinculación de tipo bidireccional entre la Universidad del Bío-Bío (en adelante UBB) y los actores presentes en el sector, con foco principal en la comunidad. Uno de los proyectos con patrocinio canadiense, se denomina: "Adaptación al cambio climático en asentamientos informales: análisis y fortalecimiento de iniciativas locales en Latinoamérica y el Caribe", cuyo objetivo es estudiar y crear condiciones para el crecimiento y la transferencia de estrategias informales de adaptación al cambio climático en contexto de vulnerabilidad y con presencia de liderazgo femenino. El otro es el Laboratorio de Gobierno Local (LabUBB), que busca la generación de un modelo de gestión de innovación pública local y con apoyo del Gobierno Regional del Biobío. El tercer proyecto, surge desde el Taller de Barrios de la Escuela de Arquitectura, al que se suma desde el 2017 a la carrera de Trabajo Social. Bajo la idea general de aprendizaje y servicio, se empuja la investigación docente, que vincula a estudiantes de diversos niveles de aprendizaje (aprox. 160 estudiantes, 10 académicos y profesionales). Los estudiantes realizan actividades en terreno y en el aula, dirigidos por los docentes y en contacto con la comunidad. En este contexto, se han generado datos de relevancia que permiten sustentar de forma exploratoria este trabajo.

Nos planteamos identificar cuáles son las temáticas que los procesos de gobernanza territorial de espacios periurbanos de metropolización reciente están enfrentando, en qué se basa su emergencia como temas relevantes y los desafíos que estos plantean. Organizamos el artículo dando cuenta en primer lugar, de los marcos teóricos a partir de los cuales comprendemos los procesos de gobernanza del espacio. En segundo lugar, explicamos brevemente la metodología de análisis utilizada en la investigación, para en tercer lugar dar cuenta de los resultados obtenidos. Finalmente, se concluye con algunas reflexiones preliminares de carácter exploratorio.

\section{Aspectos teóricos}

Para efectos de este trabajo asumimos la metropolización como un dato de contexto, como un fenómeno descrito y asumido desde el estudio de las transformaciones en las últimas décadas de las ciudades en particular y el territorio nacional chileno en general (Hidalgo y Arenas, 2009). Todos estos procesos han empujado a replantearse los modelos de planificación y análisis del desarrollo socioespacial. Emerge como tema relevante los procesos de gobernanza metropolitanas y el estudio de los conflictos socio-espaciales en el marco de la creciente urbanización de los territorios. Por ello, nos parece relevante abordar la relación entre este contexto en desarrollo (la metropolización) y los procesos de gobernanza territorial. Con ello se pretende contribuir a la comprensión de las complejidades que emergen como desafíos para el desarrollo de las comunidades afectadas. 
Desafíos para la gobernanza en espacios periurbanos metropolizados: el caso de Nonguén, en Concepción, Chile por Javier León Aravena, Felipe Saravia Cortés e Ignacio Bisbal

Grandal.http://doi.org/10.29035/pai.4.2.24

\title{
Teoría de la gobernanza
}

La gobernanza, suele abordarse a partir de dos dimensiones que pueden analizarse por separado o formar parte de un solo constructo. Nos referimos, por un lado, a una perspectiva con un énfasis organizacional y, por otro lado, a otra perspectiva que enmarca la gobernanza en la teoría democrática. Nos interesa la segunda perspectiva, que, sin ser excluyente de la primera, sitúa el aspecto organizacional como un componente que puede cobrar relevancia según el énfasis particular del estudio. Dentro del campo de la gobernanza democrática, existe en un extremo lo que se denomina una gobernanza a "secas" y, en el otro, una gobernanza con apellido (o adjetivo), indicando con ello, la orientación hacia donde se le quiere imprimir un contenido particular (Arellano, 2014). Por ejemplo: gobernanza democrática, territorial, ambiental, etc. Entendemos la gobernanza como

\begin{abstract}
"un modo postgubernamental de gobernar en numerosos asuntos públicos, que se ejerce mediante redes, sinergias y asociaciones entre actores del gobierno y actores de la sociedad económica y civil y que se considera es el proceso de gobernar apropiado para las condiciones sociales actuales, pues incrementa las capacidades, recursos y alcances de los gobiernos". (Aguilar, 2014, p.12)
\end{abstract}

Este enfoque vinculado, en principio, a la NGP (nueva gestión pública), enfatizaba los componentes de eficiencia gubernamental por sobre los aspectos normativos. En este sentido, un primer concepto de gobernanza apuntó tanto a la capacidad del gobierno, de encauzar potencialidades y recursos, como a la eficacia de la acción (Aguilar, 2006). La complejidad de esto radica en que los múltiples niveles en los que se expresa esta gobernanza, tanto hacia la sociedad como hacia la propia organización administrativa y territorial del Estado, no agota el alcance del concepto solo en la esfera de eficiencia gubernamental. Por ello, la definición no se puede quedar en la esfera institucional, sino que el análisis de la gobernanza en el territorio debe profundizar en la caracterización de la dinámica y sentido de las relaciones también entre los ámbitos públicos y privados. En este sentido, manteniendo un sentido normativo, la gobernanza puede ser operacionalizada según los énfasis que se le impregnan a los estudios particulares a los que se asocia, en unos casos apela a la conformación de redes macroestructurales, en otros a nivel local, puede haber focos temáticos amplios o específicos, pero en todos ellos se busca el análisis de la relación de intereses y su confirmación en una red decisional. Es decir, esto implica tipo de actores, calidad de relaciones y niveles territoriales/espaciales de esta gobernanza. Un modelo utilizado para este tipo de operacionalización es el RIS3 (Unión Europea, 2012), el que identifica seis esferas en las que se ubican diversos tipos de actores que podrían participar de estrate- 
gias territoriales en torno a planes de desarrollo económicos-sustentables y de especialización estratégica. Las esferas de interés son: las autoridades políticas y agencias del estado, la sociedad civil, los actores del conocimiento, inversores, empresas y alianzas internacionales.

Desde el punto de vista de la gobernanza como un ideal normativo, vinculado a la calidad de la democracia, se plantea que la buena calidad de dicha gobernanza, está asociada a las relaciones entre cultura democrática, capital social, valores de cooperación y confianza, etc. (Rodríguez, 2013). En este sentido hay enfoques que conceptualizan la gobernanza como una articulación de asuntos técnicos y políticos en vista a mejorar la calidad de vida de territorios y el abordaje no sectorializado de problemas, incluso de forma multinivel o multiescalar y flexible para realidades complejas, fomentando el diálogo entre actores con un sentido de corresponsabilidad. Por ello, una gobernanza democrática enfocada en los territorios no puede solo centrarse en los actores estatales, sino que en el sistema de actores de dicho territorio en su conjunto (Unión Europea, 2011). Dentro de este sentido estamos enmarcando el análisis del territorio Nonguén, es decir, como un espacio donde hay multiplicidad de actores, que establecen diversas formas de relación con el objetivo de conseguir desarrollo colectivo. Nos interesa estudiar y descubrir cómo es la gobernanza en el sector Nonguén, como un marco analítico y empírico, que nos permitirá indagar y caracterizar actores, sus relaciones, el sentido que estas tienen y los problemas que las determinan. En último término, cómo la gobernanza democrática es un factor coadyuvante del desarrollo de intervenciones socioespaciales institucionalizadas o no institucionalizadas en este territorio.

La gobernanza, como hemos dicho, se ha impuesto como un concepto que enfatiza el gobierno en red, basado en capital social y relaciones de confianza, como un proceso clave para determinar la calidad de las relaciones y la gobernabilidad de un territorio; excede lo meramente estatal. En definitiva, es un ideal normativo que conlleva el riesgo de obviar el hecho de que las sociedades son complejas y que en su seno coexisten conflictos que no siempre se resuelven sólo por una orientación normativa, y que ello implica que los procesos reales de negociación y regulación de conflicto resultan claves para mejorar las condiciones de la gobernanza (Arellano, 2014). Así, desde un punto de vista amplio, el concepto de gobernanza explica un ámbito importante de las relaciones de incidencia política, aquella dimensión donde existen interacciones decisionales, las que tendrán expresiones políticas en la acción directa en el territorio. Corresponde, por lo tanto, a una forma de definir las relaciones entre política y sociedad, buscando profundizar en aspectos de legitimidad, participación y gobernabilidad (Dalla-Torre, 2017). Finalmente, la gobernanza se refiere a múltiples estrategias políticas para redefinir la relación entre sociedad y Estado (Cano, 2008), en la lógica de generar cambios, procesos de incidencia y, en suma, para el territorio Nonguén, la búsqueda y consolidación de un modelo de desarrollo sustentable. 
Desafíos para la gobernanza en espacios periurbanos metropolizados: el caso de Nonguén, en Concepción, Chile por Javier León Aravena, Felipe Saravia Cortés e Ignacio Bisbal

Grandal.http://doi.org/10.29035/pai.4.2.24

\section{El espacio como producto social}

Entendemos que los procesos de gobernanza se desarrollan en torno a procesos sociales que no solo tienen lugar en el espacio, sino que son condicionados o moldeados por este. En este mismo sentido, los procesos de gobernanza abordan siempre fenómenos de carácter socioespacial, dada la indivisibilidad de estas dos dimensiones: lo social y lo espacial. Nos posicionamos entonces desde una lógica Lefebvriana que concibe el espacio como un producto social, es decir, no preexistente a la acción humana y, al mismo tiempo, no como un mero contenedor de procesos sociales, sin mayor incidencia en el devenir de estos. En el planteamien-to de Lefebvre (2013) a firma que "el espacio (social) no es una cosa entre las cosas, un pro-ducto cualquiera entre los productos: más bien envuelve a las cosas producidas y comprende sus relaciones en su coexistencia y simultaneidad" (p.129). De esta manera, las condiciones materiales de vida de los habitantes de un determina-do lugar y sus prácticas cotidianas en el espacio, así como sus representaciones mentales relativas a este, están condicionadas por el espacio mismo. Es decir, el espacio es visto entonces, como producto de relaciones sociales y al mismo tiem-po productor de estas.

Ahora bien, en el proceso de producción del espacio se producen disputas de poder respecto de la producción y del control de este, y no todos los actores implicados tienen la misma capacidad de incidencia en dicho proceso. Lefebvre (2013) plantea que en el contexto capitalista, el mayor poder de producción del espacio recae en el capital, por sobre las clases trabajadoras. En el contexto urbano, este se expresa en el desarrollo inmobiliario que presiona por avanzar hacia formas del uso del suelo que se adecúen a sus propios intereses empresariales (Panez, 2010).

En suma, nos posicionamos desde una perspectiva del espacio como una dimensión abarcativa de la realidad social, multidimensional-considera de forma imbricada aspectos materiales, subjetivos y sociales-, dinámica e histórica (Lefebvre, 2014). En este sentido, entendemos que la realidad socioespacial actual de Nonguén, se asienta en su trayectoria histórica, marcada por su inserción en el proceso de metropolización del gran Concepción, y su incorporación en la mancha urbana durante las últimas décadas. 


\section{Aspectos metodológicos}

El análisis de la gobernanza, implica una identificación detallada de actores, que es un ámbito que el de estudio del sector debe profundizar. Por ahora, no contamos con un catastro ni análisis detallado de las composiciones de la red de actores ni sus características y solo podemos dar cuenta de una noción bastante general del tipo de relación existente. Tenemos en este trabajo insumos relativos a los problemas y desafíos que líderes de asociaciones locales plantean y que nos permite sentar las bases para su posterior profundización. En este sentido el trabajo es un avance de investigación. Sin embargo, nos parece valioso el panorama que con los datos disponibles se presenta para el análisis.

La investigación que desarrollamos tiene, por lo tanto un carácter exploratorio, y se basa en una metodología cualitativa (Gibss, 2012). Utilizamos principalmente como fuente de información los contenidos provenientes de una jornada de trabajo con dirigentes de organizaciones vecinales y comunitarias de Nonguén, realizada el día sábado 20 de Octubre del año 2018. En esta se desarrolló un trabajo de taller para reflexionar de forma colectiva acerca de los asuntos que los dirigentes, líderes y vecinos del sector consideran relevantes para el desarrollo de este. Se conformaron 9 grupos de aproximadamente 7 personas cada uno, los que trabajaron en torno a tres preguntas: ¿qué de valor se ha perdido en el sector Nonguén?, ¿qué del sector Nonguén de hoy quisiera conservar, cambiar o recuperar?, y ¿qué quisiera que hubiese en el sector Nonguén que hoy no hay? Las respuestas a estas fueron registradas y sintetizadas en informes a cargo de facilitadores, que fueron principalmente académicos de las Escuelas de Arquitectura y Trabajo Social de la Universidad del Bío-Bío.

Para el análisis se utilizó el software Atlas.ti. Se codificaron los 9 informes de la jornada de trabajo con dirigentes, identificando los principales tópicos por estos relevados en relación con lo que consideran se ha perdido en el sector, aquello que quieren conservar, cambiar o recuperar, y en cuanto a los sueños y visiones de futuro territoriales. Esto es presentado de forma descriptiva buscando encontrar las principales tendencias en los relatos.

Se aplicó un análisis categorial temático simple, con una codificación apriorística según preguntas indicadas, y una codificación abierta para la identificación de los tópicos relevados por los entrevistados. Se elaboró una tabla de co-ocurrencia de códigos para determinar contenidos de las respuestas a las preguntas planteadas. Esta herramienta del software Atlas.ti arroja un valor que fluctúa entre 0 y 1 , siendo los valores más cercanos a 1 , aquellos que indican relaciones más intensas de co-ocurrencia. 
Desafíos para la gobernanza en espacios periurbanos metropolizados: el caso de Nonguén, en Concepción, Chile por Javier León Aravena, Felipe Saravia Cortés e Ignacio Bisbal

Resultados

\section{Objetos de la gobernanza territorial}

Identificamos en primer lugar aquellos temas que podrían constituir el objeto de la gobernanza territorial, a partir del relato de los dirigentes vecinales de Nonguén. La tabla 1, muestra en las columnas cuatro tipos de grandes temas, agrupables en dos conjuntos: aquellos que se centran en aspectos positivos de Nonguén, y otros que se centran más bien en aspectos que deben ser modificados. En el primer conjunto distinguimos entre aquello positivo que se ha perdido con el paso del tiempo de aquello positivo con que se cuenta en la actualidad y que debe ser conservado. En el segundo conjunto distinguimos aquellos elementos que en la actualidad deben ser modificados, y aquellos elementos que constituyen las visiones de futuro para Nonguén. Estos cuatro temas (códigos apriorísticos) se cruzan con contenidos que fueron producto de una codificación abierta, y se muestran en colores más oscuros las asociaciones más intensas, producidas por co-ocurrencia de códigos. 
Tabla 1. Relación entre tópicos emergentes y temas planteados a dirigentes vecinales*.

\begin{tabular}{|c|c|c|c|c|}
\hline & Pérdidas & $\begin{array}{l}\text { Aspectos que } \\
\text { se desean } \\
\text { conservar }\end{array}$ & $\begin{array}{l}\text { Aspectos que } \\
\text { se desean } \\
\text { cambiar }\end{array}$ & $\begin{array}{l}\text { Deseos para } \\
\text { Nonguén }\end{array}$ \\
\hline Alcoholismo & 0 & 0 & 0,07 & 0 \\
\hline Carabineros & 0 & 0,06 & 0,06 & 0,27 \\
\hline Contacto con la naturaleza & 0,38 & 0,19 & 0,12 & 0,27 \\
\hline Cuidado del entorno natural & 0,14 & 0,16 & 0,23 & 0,33 \\
\hline Delincuencia & 0,31 & 0 & 0,16 & 0,06 \\
\hline Deporte & 0 & 0,06 & 0,06 & 0,18 \\
\hline Desarrollo inmobiliario & 0,22 & 0,04 & 0,17 & 0,29 \\
\hline Droga & 0,1 & 0 & 0 & 0,1 \\
\hline Espacios de recreación & 0,15 & 0 & 0,05 & 0,25 \\
\hline Estudiantes universitarios & 0 & 0,07 & 0 & 0 \\
\hline Fiesta pública & 0,44 & 0,04 & 0,04 & 0,28 \\
\hline Fiscalización / multas & 0 & 0 & 0 & 0,11 \\
\hline Inclusividad & 0 & 0 & 0 & 0,22 \\
\hline Infraestructura urbana pública & 0,08 & 0,14 & 0,14 & 0,65 \\
\hline $\begin{array}{l}\text { Intercambios económicos } \\
\text { informales }\end{array}$ & 0,33 & 0 & 0 & 0 \\
\hline Liderazgo femenino & 0 & 0,07 & 0,07 & 0 \\
\hline Liderazgo y participación juvenil & 0,18 & 0 & 0 & 0,18 \\
\hline Liderazgos vecinales & 0 & 0,25 & 0,05 & 0,07 \\
\hline $\begin{array}{l}\text { Nuevos barrios / Nuevos } \\
\text { vecinos }\end{array}$ & 0,1 & 0 & 0 & 0,1 \\
\hline Paisaje bello & 0,11 & 0 & 0 & 0 \\
\hline Participación ciudadana & 0,22 & 0 & 0 & 0 \\
\hline Patrimonio & 0,07 & 0,25 & 0 & 0,07 \\
\hline Relaciones intergeneracionales & 0,22 & 0 & 0 & 0 \\
\hline Relación con UBB & 0 & 0,13 & 0 & 0,18 \\
\hline Riesgos socioambientales & 0 & 0 & 0,07 & 0,1 \\
\hline $\begin{array}{l}\text { Sentido de comunidad / } \\
\text { prácticas comunitarias }\end{array}$ & 0,62 & 0,12 & 0,24 & 0,17 \\
\hline Servicios comerciales / privados & 0 & 0 & 0,05 & 0,5 \\
\hline Tráfico de camiones & 0 & 0 & 0,07 & 0 \\
\hline Tranquilidad & 0,33 & 0 & 0 & 0 \\
\hline Transporte público & 0 & 0 & 0,07 & 0,1 \\
\hline Turismo & 0 & 0 & 0 & 0,22 \\
\hline
\end{tabular}

*0,1 a 0,19 = relaciones débiles; 0,2 a 0,29 = relaciones medias; 0,3 a 0,39= relaciones medias altas; 0,4 o más $=$ relaciones fuertes.

Fuente: elaboración propia. 
Desafíos para la gobernanza en espacios periurbanos metropolizados: el caso de Nonguén, en Concepción, Chile por Javier León Aravena, Felipe Saravia Cortés e Ignacio Bisbal

Grandal.http://doi.org/10.29035/pai.4.2.24

\section{Elementos perdidos}

Los aspectos que más se mencionan en relación con las pérdidas, son el sentido de comunidad y prácticas comunitarias asociadas, especialmente fiestas públicas y comunitarias, así como la presencia de intercambios económicos informales. Se destaca también la pérdida de contacto directo con la naturaleza, y la pérdida de tranquilidad, asociado al aumento de la delincuencia en el sector.

En cuanto al sentido de comunidad, los dirigentes vecinales indican que se han perdido ciertas prácticas comunitarias como la realización de festivales o de carnaval en el sector. Cuentan que la realización periódica de esta actividad los unía. Se cerraba la calle Nonguén y se utilizaban las canchas para los eventos, y la construcción y muestra de carros alegóricos. Estas prácticas perdidas son referidas como parte una dimensión espiritual del ser comunidad. Se visualiza a dicha comunidad perdida como una familia ampliada en que todos los vecinos participaban. Esta se asentaba en la existencia de una organización vecinal fuerte, quienes potenciaban también otras actividades como la fiesta de la primavera y ramadas. Hay un aspecto en los relatos de lo perdido que dice relación con un pasado más rural o campesino, con presencia de ganado, carreras de caballo, prácticas de cultivos y trueque de mercancías. Los habitantes entrevistados confirman la pérdida de este sentido de comunidad, y lo asocian en parte, a la presencia de nuevos conjuntos residenciales que no comparten la historia e identidad común, propia de las personas que han residido en el sector desde hace varias décadas, y que tienen prácticas de conmutación que hacen que no tengan una presencia permanente.

Por otro lado, los dirigentes también asocian la pérdida del sentido comunitario al golpe militar "que sectorizó toda el área", es decir, que dividió la organización vecinal previamente existente, como ocurrió en Chile en general, y que ha redundado en una merma del poder vecinal y del sentido de cooperación (Letelier, Micheletti, y Vanhulst, 2016).

Además, existe preocupación porque todos estos procesos han implicado una menor participación ciudadana en el sector con especial pérdida de participación de jóvenes. En efecto, el sentido de comunidad perdido afecta también las relaciones intergeneracionales, lo que implica que no hay una relación intensa entre adultos, adultos mayores y jóvenes. Algo que puede estar ocultando esta percepción es la desconexión de cierta dirigencia más tradicional con nuevas formas de asociatividad que se mueven por el sector, pero que no dialogan necesariamente con la estructura más consolidada, en este sentido la gobernanza institucional podría requerir ajustes con la apertura a nuevas formas de participación, la validación y reconocimiento de otros tipos de actores. 
El contacto con la naturaleza apunta a una vida cotidiana con una fuerte relación con los espacios disponibles que el sector tuvo o que existen pero se ven más lejanos. Se relatan paseos bajo árboles, piscinas, paseos por el estero o los cerros. Se mencionan también otras actividades relativas a la producción de la vida diaria, como abastecerse de agua, combustible, producir sus propios alimentos. Todo esto es referido desde una perspectiva de añoranza, tanto por dirigentes como por el resto de entrevistados. Hoy la relación con el estero es más bien escasa, se asocia a la presencia de microbasurales, y está caracterizada por una sensación de temor vinculada a la delincuencia y consumo de drogas.

En suma, todas las pérdidas se relacionan a problemáticas actuales que se perciben como consecuencias negativas, tales como la delincuencia, el desarrollo inmobiliario que implica más necesidades y nuevos vecinos, o la presencia de droga en algunos sectores.

\section{Aspectos que se desean conservar}

Del análisis de las pérdidas se identifican ideas fuerza que son importantes para la ratificación de un diagnóstico territorial. Estas ideas se configuran como recursos potenciales materializados en cuestiones tangibles e intangibles que se deben conservar, aun cuando algunos de estos componentes están afectados por las pérdidas analizadas previamente.

Un aspecto destacado es la mantención de los liderazgos vecinales existentes, que si bien es cierto se entienden dentro de una crisis de participación, son aún una fortaleza. Aquellos dirigentes que han formado parte de las luchas y han abogado por el mejoramiento de las condiciones del sector, hoy pese a ser un grupo de edad mayor, son un capital social importante y canal claro de comunicación entre las diversas instituciones que se insertan el sector. Desde este punto de vista los líderes que hoy existen son también un patrimonio, y forman la base más potente para el desarrollo de la gobernanza territorial.

Hay otro aspecto también de tipo patrimonial, y que dice relación con la tradición campesina o rural, en este sentido tanto el medio natural, como el espacio sociocultural, las identidades, tradiciones e historias forman parte de un rico patrimonio tangible e intangible que la comunidad desataca como algo a conservar. En este sentido, se hacen esfuerzos por generar plataformas de apoyo: existe una biblioteca y radio comunitaria, así como proyectos para la recuperación de un silo considerado como un elemento patrimonial, y la valorización de la reserva, entre otros. 
Desafíos para la gobernanza en espacios periurbanos metropolizados: el caso de Nonguén, en Concepción, Chile por Javier León Aravena, Felipe Saravia Cortés e Ignacio Bisbal

Grandal.http://doi.org/10.29035/pai.4.2.24

Finalmente los elementos mencionados como pérdidas, en la perspectiva de conservar o recuperar, ofrecen una conversión de debilidades en potencialidades. Por ejemplo: si bien es cierto se ha perdido el sentido de comunidad desde el punto de vista de una perspectiva de totalidad del sector, es un aspecto conservar aun en su condición disminuida, y potencialmente aparece como un factor a acrecentar. En este sentido, los líderes locales si bien es cierto se pronuncian con mucha fuerza respecto a las pérdidas y problemas del sector, establecen como parte de la misión que los inspira, realizar acciones para conservar lo existente y potenciar lo que queda. Lo mismo sucede con el entorno y cuidado de la naturaleza.

\section{Aspectos que se desean cambiar}

Lo anterior conlleva al propósito más concreto y claro, de cambio positivo en aquellos elementos que se sienten como perdidos o casi perdidos. Implica poner el acento en la fuerza social e institucional capaz de generar los cambios requeridos. Por ejemplo, el sentido de comunidad y las prácticas comunitarias perdidas o disminuidas, pueden mejorar incorporando las nuevas realidades que enfrenta el sector. Si se modifica la falta de participación de algunos grupos, se debería necesariamente abrir el abanico de posibilidades de participación. Para ello las claves de una historia de recuerdos de acción colectivas son relevantes. Es la memoria e identidad que serviría de base para impulsar un cambio. Sin embargo, el resultado no será el retorno de la misma comunidad rural que existía en el pasado, sino una nueva comunidad de tipo urbana, pero con un marco de naturaleza y perspectivas de sustentabilidad que pueden ser compatibles en lo simbólico con el pasado rural; ya no estará la Chacra Castellón (lugar donde los lugareños intercambiaban productos), pero puede haber una red de huerteros que intercambien como lo hicieron en otra época.

Es decir, se plantea que el proceso de urbanización debe ser realizado de manera tal que se mantenga e incremente el contacto con la naturaleza y el cuidado de esta, a la vez que favorezca el sentido de comunidad, y las prácticas comunitarias. Ello podría incidir en cambiar un crecimiento no regulado de viviendas o proyectos inmobiliarios, que amenaza sectores ambientales valiosos, como humedales, y que da cuenta de una regulación o fiscalización en el uso de suelos públicos y privados que debería ser mejorada. En este sentido aparece lo que se desea cambiar, como los aspectos que se deberían rescatar o reformular de modo de neutralizar su sentido asociado a pérdidas.

Especial sentido tiene lo relativo al entorno natural. Se desea recuperar el contacto con la naturaleza, cambiar la situación de lejanía o poco contacto que hay con el entorno natural. Pero se deben realizar acciones concretas para disfrutar el estero y no verlo como una amenaza, para aprovechar la reserva como un es- 
pacio cercano y que además conecta a la ciudad con el sector, construir formas de acceder a los cerros y miradores naturales que ofrecen vistas privilegiadas. En suma, se busca relacionarse de modo no invasivo con la flora y fauna del sector.

En particular, se desea cambiar el tipo de desarrollo inmobiliario, de uno invasivo y sectorizado a otro más armónico y de respeto al medio ambiente. Se desea un cambio en la situación de delincuencia, para lo cual existen muchas expectativas en el cuartel policial que se ha anunciado y por otro en potenciar los factores protectores comunitarios. Adicionalmente, se desea cambiar y mejorar la infraestructura, los servicios, la movilidad del sector, pues hay ciertos trámites y gestiones que los habitantes del sector no pueden concretar cerca de sus domicilios, debiendo desplazarse a otros sectores de la ciudad. En síntesis, de estos aspectos emerge una agenda de trabajo para la transformación socio-espacial del sector.

\section{Sueños territoriales}

Los sueños territoriales por parte de los dirigentes vecinales giran fuertemente en torno a la infraestructura urbana y la relación que esto tiene con el desarrollo de servicios comerciales asociados a un proceso de mejoramiento económico local. Existe la percepción de que este elemento de expresión material mejorado puede provocar un gran cambio en el sector. Esto no se concibe disociado del ejercicio de la organización y la participación ciudadana, ya que se percibe que el sentido de comunidad, informado como debilitado, podría fortalecerse si se produce un entorno urbano que lo haga factible.

En concreto, existen expectativas en mejoramiento de calles, de corredores urbanos, de conectividad para salir de Nonguén por otras vías. El mejoramiento de puentes es importante para la movilidad cotidiana de un sector que aún no está integralmente conectado en sus diversos subterritorios. Dentro de la infraestructura pública está lo relativo a implementar un centro cívico, que expresa la idea de una zona que permita la instalación de servicios que hoy no tienen presencia en el sector, tales como bancos, supermercados, oficinas públicas en general. En definitiva, la infraestructura urbana en general operaría aquí en su dimensión detonante más precisa, pues claramente el sueño de un sector con sentido de comunidad, tranquilo, conectado con el ambiente pasa por un mejoramiento de condiciones materiales generales. Dicha visión es compartida por los habitantes de Nonguén en general.

Ahora bien, este sueño de una urbanización mejorada no se contrapone a la ya mencionada necesidad de mayor contacto y cuidado de la naturaleza. El valor que tiene el entorno natural, va desde la presencia de la reserva Nonguén, hasta espacios más cercanos dependiendo el área de habitación. Hay personas que va- 
Desafíos para la gobernanza en espacios periurbanos metropolizados: el caso de Nonguén, en Concepción, Chile por Javier León Aravena, Felipe Saravia Cortés e Ignacio Bisbal

Grandal.http://doi.org/10.29035/pai.4.2.24

loran el cerro y su vegetación, otras los humedales, otras valoran el estero aunque de diferente forma, otras valoran los animales y la tranquilidad asociada a dicha naturaleza. Se debería trabajar entonces para impulsar un desarrollo inmobiliario sustentable y controlado. Un aspecto interesante que aparece en este proceso es el relativo concebir un rol de Nonguén en los circuitos turísticos regionales o nacionales. Los dirigentes lo asocian al potencial natural y ambiental del sector, con un vínculo a actividades económicas propias de Nonguén, como restaurantes, actividades culturales, venta de productos hortícolas, entre otros.

Finalmente, hay aspectos que se deben potenciar, como el deporte, que cuenta con instalaciones relativamente nuevas en el sector y otras por instalar como el polideportivo en los terrenos de la UBB. Por otro lado, mejorar el liderazgo juvenil y la participación ciudadana en general. Todo ello con vista a recuperar el sentido de comunidad perdido pero recuperable. Desde el punto de vista urbano, se plantea mejorar el transporte público y la relación con nuevos barrios. Por último, también es importante tener presente los riesgos socioambientales del sector para tener estrategias de abordaje claras (inundaciones, incendios, derrumbes, etcétera).

\section{Actores implicados en los procesos de gobernanza territorial de Nonguén}

En primer lugar, podemos constatar que el sector posee al menos una red de liderazgo institucionalizado con incidencia respecto a proyectos e iniciativas que se deben realizar en el sector. De hecho, para convocar a la reunión de la que surgen elementos para este análisis, se debió contactar a la mesa de asociaciones que está compuesta por a lo menos veinte organizaciones de diversa naturaleza. Aunque predominen las asociaciones vecinales tradicionales, existen otras de naturaleza diversa, tales como: grupos ambientales, comités barriales, grupos culturales, etcétera. Del mismo modo, se aprecia la presencia de instituciones locales proveedoras de servicios y del desarrollo de políticas públicas, tales como las policías (uniformada y civil). En este sentido, Carabineros de Chile alcanza una notoria presencia en los discursos. El municipio, es otro de los actores que se mencionan, pero este materializa su presencia a través de diversos departamentos (salud, educación, vivienda, entre otros). Cada unidad municipal tiene por sí misma la calidad de actor del territorio. Por otro lado, la UBB aparece evidentemente como un actor relevante dentro del sector y más bien se espera un mayor compromiso de esta entidad universitaria. Además, se identifican actores del mundo privado tales como: empresas de construcción e inmobiliarias, y servicios como restaurantes, transporte, entre otros. 
Faltaría potenciar y reconocer otras formas de participación, asociadas a grupos de edades diferentes, lo que implicaría trabajar con una estrategia intergeneracional puesto que, como es común, las asociaciones tradicionales están compuestas principalmente por personas mayores.

En definitiva, Nonguén aglutina una diversidad de actores que lo cualifican potencialmente como un buen sector para el desarrollo de estrategias de gobernanzas en territorios sustentables. Habría que analizar con más detalle aspectos como la densidad de relaciones, niveles de confianza, redes de actores formales e informales, las perspectivas de género en los procesos asociativos, entre otras variables importantes. Lo interesante de este punto son las posibilidades que los actores tendrían para plantearse formas de gobernanza adecuadas a los cambios que están experimentando. Es decir, existen recursos sociales para plantearse estrategias de desarrollo de asociatividad, y el sector posee referentes materiales y simbólicos potentes para construir formas de autogestión del desarrollo en colaboración con otros actores en una perspectiva multiactor y multinivel.

\section{Reflexiones finales}

El sector Nonguén, se puede enmarcar con fines analíticos operativos, en lo que se ha denominado gobernanza territorial (Unión Europea, 2011), territorio compuesto por diversidad de actores locales de la sociedad civil e institucionales, de diversos niveles de la gestión pública. Entre ellos se encuentran: juntas de vecinos, radio y biblioteca comunitaria, policías, municipio, universidades, etc. Estos tienen múltiples desafíos para generar calidad de relaciones democráticas, confianza y legitimidad en las decisiones que los afectan. Dichas decisiones están relacionadas a las diversas problemáticas que ellos expresan y que involucran aspectos de identidad y experiencias, anhelos y expectativas sobre la forma en que se puede mejorar la calidad de vida de sus habitantes. En cuanto a tipos de espacios, tangibles o intangibles, existen determinantes que definen grupos de interés, algunos transversales o temáticos (como grupos etarios, ambientales, culturales), otros grupos están relacionados a micro territorios dentro del marco amplio del sector, con temas relacionados a infraestructura vial y movilidad, habitabilidad, seguridad pública, etc.

Entre los temas relevantes, está el relativo a la sensación de pérdida de confianza y lazos comunitarios, esto genera la percepción de que hay habitantes que no se interesan por la historia, el presente y futuro del sector. En este sentido es necesario ahondar en el estudio de la calidad de las relaciones y el sentido que estas tienen. Del mismo modo que analizar con más detalles la opinión de otros habitantes y las posibles nuevas formas de organización, por ejemplo las redes sociales. 
Desafíos para la gobernanza en espacios periurbanos metropolizados: el caso de Nonguén, en Concepción, Chile por Javier León Aravena, Felipe Saravia Cortés e Ignacio Bisbal

Grandal.http://doi.org/10.29035/pai.4.2.24

Existe el desafío de profundizar este trabajo con mayor información y claridad respecto a la identificación de todos los actores de este territorio, al menos en un nivel mucho más completo respecto a las temáticas y desafíos planteados. Sabemos que hay organizaciones tradicionales y emergentes pero no sabemos cuántas y su distribución territorial, y tampoco tenemos un conocimiento detallado del tipo de iniciativas que estos autogestionan, necesitamos profundizar en las relaciones y su calidad.

En suma, este trabajo buscó identificar cuáles son las temáticas que los procesos de gobernanza territorial que debe abordar Nonguén, en tanto espacio periurbano de metropolización reciente. Al respecto se encontró que este sector ha perdido, a lo largo del tiempo, distintos elementos que eran valorados y que hoy se añoran. Como contracara, los resultados muestran que los sueños territoriales están depositados principalmente en el mejoramiento en calidad y cantidad de elementos de la urbanización. Ello podría ser considerado contradictorio, en la medida que pareciera ser que ha sido precisamente el proceso de urbanización e incorporación a la metrópolis, uno de los principales factores explicativo de la pérdida de los elementos valiosos ya mencionados. Dicha aparente paradoja puede ser entendida distinguiendo la posibilidad de desarrollar formas de urbanización que mantengan aquello que los habitantes consideran valioso, e inclusive lo potencien. En este punto cabe destacar la importancia de las fiestas públicas como dispositivo de intervención (Letelier y Rasse, 2016), por ello el potencial que tienen de convertirse en factor promotor de cambios.

La evidente tensión entre pasado rural y presente urbano va más allá de los efectos y problemas que los actores analizados describen. Hay procesos más profundos que configuran una cierta discontinuidad entre el tipo de comunidad rural añorada y el presente metropolitano al que se enfrenta la población. Particularmente complejo es que la metropolización de esta ciudad no se basa en una organicidad funcional, sino que también en el propio proceso de urbanización y exclusión que han sido parte del modelo que prima en la realidad chilena. Eso implica que a diferencia de otras metrópolis que han resuelto ya a mediados del siglo XX diversos problemas derivados de la condición metropolitana, tales como la disposición de agua potable, sistemas de viviendas, transporte, contaminación, etcétera, esta metrópoli toma conciencia general de su condición sin tener aún consenso sobre cómo abordar la movilidad de las personas, la organización funcional de los servicios públicos, la gestión de los residuos y los riesgos, entre otros. Para abordar esos nuevos desafíos que enfrenta a Nonguén con su particularidad espacial, es que se requiere de una gobernanza territorial definida y validada por los actores de este territorio. Para ello deberían dar un salto desde formas tradicionales que al parecer aún dominan las formas de articulación decisional a nuevas formas post gubernamentales (Aguilar, 2014). En este proceso, actores 
como la universidad, nuevos tipos de asociaciones, la interacción con el mundo privado presente y omnipresente en el sector, en otras categorías de actores, que se impliquen en la construcción socio espacial bajo una gobernanza democrática, es esencial.

Finalmente, la universidad y las acciones de vinculación están teniendo buenos resultados respecto a establecer un diálogo abierto con la comunidad hasta ahora convocada, puesto que en el marco de los proyectos mencionados que sustentan este trabajo, se han realizado más actividades de diálogo, diagnóstico e investigación que permitirán mejorar los conocimientos relacionados.

Agradecimientos: Agradecemos el financiamiento otorgado por parte del proyecto "Climate change adaptation in informal settings in Latin America and the Caribbean: understanding and reinforcing bottom-up initiatives", financiado por la organización canadiense "International Development Research Centre" (IDRC), y al grupo de vecinos, estudiantes y académicos que hacen posible el contar con información del sector. 
Desafíos para la gobernanza en espacios periurbanos metropolizados: el caso de Nonguén, en Concepción, Chile por Javier León Aravena, Felipe Saravia Cortés e Ignacio Bisbal

Grandal.http://doi.org/10.29035/pai.4.2.24

\section{Referencias}

Aguilar, I. (2014). Las dimensiones y los niveles de la gobernanza. Cuadernos de Gobierno y Administración Pública, 1(1), 11-36.

Aguilar, I. (2006). Gobernanza y gestión pública. México: FCE.

Arellano, D. (2014). ¿ ¿Uno o varios tipos de gobernanza? Más allá de la gobernanza como moda: la prueba del tránsito organizacional. Cuadernos de Gobierno y Administración, 1(2), 117-137. doi:10.5209/rev_CGAP.2014.v1.n2.47538

Cano, L. (2008). La participación ciudadana contra la corrupción: en las políticas públicas de lucha respondiendo a la lógica de gobernanza. Estudios Públicos, 33, 147-177. doi:10.13140/RG.2.1.1909.8000

Dalla-Torre, M. A. (2017). Gobernanza territorial y planes de ordenamiento territorial: el caso de la provincia Mendoza, Argentina. Bitácora Urbano Territorial, 27(1), 47-54. doi:10.15446/bitácora.v27n1.47597

Diario Concepción. (Septiembre, 2018). Valor del suelo en Concepción se ha duplicado en los últimos diez años. Rescatado de: https://assets. diarioconcepcion.cl/2018/09/22-09-2018.pdf

Gibss, G. (2012). El análisis de datos cualitativos en investigación cualitativa. Madrid: Ediciones Morata.

Hidalgo, R., y Arenas, F. (2009). Del país urbano al país metropolitano. Transformaciones recientes en las ciudades chilenas. En R. Hidalgo, C. de Mattos, y F. Arenas (Eds.), Chile: del país urbano al país metropolitano (pp. 9-29). Santiago: PUC.

Lefebvre, H. (2014). El pensamiento marxista y la ciudad. Ciudad de México: Ediciones Coyoacán.

Lefebvre, H. (2013). La producción del espacio. Madrid: Capitán Swing.

Letelier, F., Micheletti, S., y Vanhulst, J. (2016). Prácticas instituyentes en el espacio vecinal: el barrio como un común. Polis, 15(45), 105-119.

Letelier, F., y Rasse, A. (2016). La fiesta pública como dispositivo de animación y resignificación del espacio público vecinal: cuatro casos en la ciudad de Talca. Sociétés, 132(2), 99-112. doi:10.3917/soc.132.0099

MINSAL. (2017). Cesfam Villa Nonguén de Concepción oficializó Acreditación en Calidad. Consultado el 25/11/2018 en: https://www.minsal.cl/cesfam-villanonguen-de-concepcion-oficializo-acreditacion-en-calidad/ 
Panez, A. (2010). La disputa por la ciudad: la importancia de una perspectiva urbana crítica en trabajo social. O Social em Questão, 13(24), 55-68.

Rodríguez, P. (2013). El capital social como factor de innovación y desarrollo empresarial en Andalucía. Sevilla: Consejo Económico y Social de Andalucía.

Unión Europea. (2011). Ciudades del Mañana. Retos, visiones y caminos a seguir. Bruselas: UE.

Unión Europea. (2012). Guide to research and innovation strategies for smart specialisations (RIS 3). Luxembourg: Publications Office of the European Union.

Dirección de correspondencia:

Javier León Aravena. Trabajador Social. Doctor en Ciencia Política. Académico de la Universidad del Bío-Bío, Concepción, Chile.

Contacto: jleon@ubiobio.cl

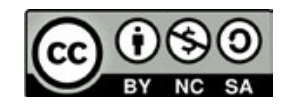

Esta obra se encuentra bajo una Licencia de Creative Commons Reconocimiento-NoComercial-Compartirlgual 4.0 Internacional 